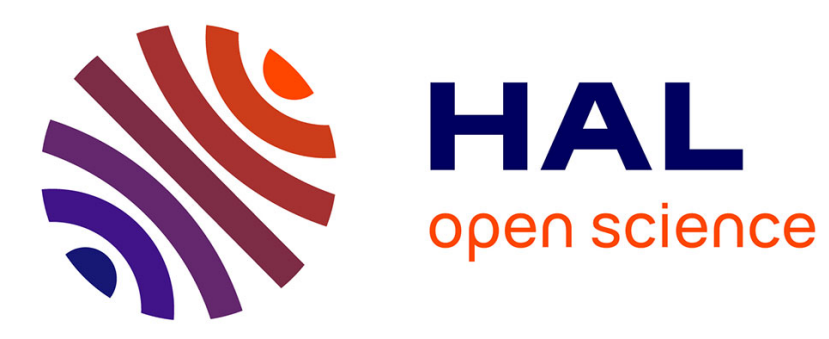

\title{
Des partis et des jeunes. Les droites juvéniles dans l'Italie de Berlusconi
}

\author{
Stéphanie Dechezelles
}

\section{To cite this version:}

Stéphanie Dechezelles. Des partis et des jeunes. Les droites juvéniles dans l'Italie de Berlusconi. Histoire@Politique: revue du Centre d'histoire de Sciences Po, 2008, Les jeunes, sujets et enjeux politiques (France, XXe siècle), 4, pp.13. 10.3917/hp.004.0014 . halshs-00326544

\section{HAL Id: halshs-00326544 https://shs.hal.science/halshs-00326544}

Submitted on 19 Jan 2022

HAL is a multi-disciplinary open access archive for the deposit and dissemination of scientific research documents, whether they are published or not. The documents may come from teaching and research institutions in France or abroad, or from public or private research centers.
L'archive ouverte pluridisciplinaire HAL, est destinée au dépôt et à la diffusion de documents scientifiques de niveau recherche, publiés ou non, émanant des établissements d'enseignement et de recherche français ou étrangers, des laboratoires publics ou privés. 
Stéphanie Dechezelles, « Des partis et des jeunes. Les droites juvéniles dans l'Italie de Berlusconi », Histoire@Politique. Politique, culture, société, N²4, janvier-avril 2008, www.histoire-politique.fr

\title{
Des partis et des jeunes. Les droites juvéniles dans l'Italie de Berlusconi
}

\author{
Stéphanie Dechezelles
}

Fruits d'un lent mais progressif effritement des bases électorales des partis piliers de la Première République (1948-1992) et d'une forte délégitimation de leurs élites impliquées dans le système de corruption généralisé (Tangentopoli), d'importants bouleversements ébranlent l'échafaudage partisan italien au cours de la décennie 1990. Par la mise en cause directe de la classe politique dans des enquêtes puis des procès, les juges de l'opération Mani pulite en appellent à une moralisation de la vie politique nationale. Très populaire alors, l'argument est repris avec force par les représentants de la majorité comme les leaders de l'opposition, qui voient là s'ouvrir l'espoir, jusqu'alors déçu, de conquête du pouvoir. Le « changement » ou le besoin d'hommes « neufs » deviennent des slogans communs à différents acteurs politiques qui, grâce à des défections et des recompositions d'alliances, peuvent envisager le renversement des logiques antérieures d'accès au pouvoir. Conçu comme un moyen de « moraliser » la vie politique en évitant les dérives du régime antérieur, le système électoral est modifié. Ainsi, à un mode de scrutin proportionnel favorisant les gouvernements pluripartites placés invariablement sous le contrôle hégémonique de la Democrazia Cristiana et, dans une moindre mesure, du Partito Socialista italiano depuis les débuts de la République ${ }^{1}$, succède un mode de scrutin mixte (aux trois quarts majoritaire et proportionnel) pour les élections législatives (Chambre des députés et Sénat).

Ces changements provoquent une refonte importante de l'offre partisane: Democrazia Cristiana et Partito Comunista italiano disparaissent, tandis que de « nouveaux » acteurs politiques émergent et que l'ensemble des forces politiques se bipolarise. Aux législatives de mars 1994, on compte trois coalitions: le Pôle progressiste autour des forces de gauche et d'une petite partie du centre; le Pacte pour l'Italie autour du Partito Popolare et du Patto de l'ex-démocrate chrétien Marco Segni ; le Pôle des libertés et du bon gouvernement réunissant les partis de droite et d'extrême droite autour de Silvio Berlusconi. Mais lors des différentes élections législatives suivantes, en 1996 puis 2001, ce sont deux coalitions qui s'opposent. La coalition de "gauche», l’Ulivo, menée par Romano Prodi se retrouve face à la coalition de « droite » menée par Silvio Berlusconi (Forza Italia et Alleanza Nazionale d'abord, puis Forza Italia, Alleanza Nazionale, Lega Nord, et les ex-démocrateschrétiens Centro cristiano Democratico et Cristiani Democratici Uniti). L'un des bouleversements majeurs sur la scène politique italienne est donc le succès d'organisations partisanes de «droite», pour l'une jusque-là exclue du pouvoir (la post-fasciste Alleanza Nazionale de Gianfranco Fini) ${ }^{2}$, pour une autre insuffisamment forte pour accéder au gouvernement national (l'autonomiste Lega

\footnotetext{
${ }^{1}$ Silvio Lanaro, Storia dell'Italia repubblicana, Venise, Marsilio, 1992.

2 Piero Ignazi, Il Polo escluso. Profilo del Movimento sociale italiano, Bologne, Il Mulino, 1989.
} 
Stéphanie Dechezelles, « Des partis et des jeunes. Les droites juvéniles dans l'Italie de Berlusconi », Histoire@Politique. Politique, culture, société, N²4, janvier-avril 2008, www.histoire-politique.fr

Nord d'Umberto Bossi) ${ }^{3}$ et pour une autre encore très récemment créée (la double héritière socialiste et démocrate-chrétienne Forza Italia de Silvio Berlusconi) ${ }^{4}$. Bénéficiant d'un climat défavorable aux « héritiers recyclés » du système politique précédent, discrédités par les scandales politico-affairistes, ces partis et leur leader s'affirment comme forces légitimes de gouvernement ${ }^{5}$.

Ce contexte de transition partisane offre à l'observateur une très bonne occasion d'étudier les effets de fortes transformations et la pratique du pouvoir par de « nouveaux » acteurs. De la même façon, parce que les règles du jeu se trouvent en partie perturbées ${ }^{6}$, voire changées, il semble opportun de se pencher sur ces organisations situées à droite de l'échiquier politique afin de comprendre, de l'intérieur, la manière dont les acteurs de ces partis perçoivent de telles mutations. Enfin, l'arrivée sur la scène politique de nouveaux entrepreneurs politiques, tentant d'établir leur légitimité sur la rupture avec les modes passés de pratique du pouvoir mais aussi de recrutement militant ${ }^{7}$, permet d'observer in vivo les voies d'accès à l'engagement et les effets de ces transformations. Afin de prendre la mesure de ces mutations, un éclairage peut être porté sur les organisations de jeunesse de chacun des principaux partis de la Casa delle Libertà, i.e. Forza Italia Giovani pour Forza Italia, Azione Giovani pour Alleanza Nazionale et le Movimento Giovani Padani pour la Lega Nord.

\begin{tabular}{|c|c|c|c|}
\hline Parti politique & Forza Italia & Lega Nord & Alleanza Nazionale \\
\hline $\begin{array}{l}\text { Organisation de } \\
\text { jeunesse }\end{array}$ & $\begin{array}{l}\text { Forza Italia Giovani - Giovani } \\
\text { per la Libertà }\end{array}$ & Movimento Giovani Padani & Azione Giovani \\
\hline $\begin{array}{c}\text { Obligation de s'inscrire } \\
\text { au parti }\end{array}$ & Oui & Non & Non \\
\hline $\begin{array}{l}\text { Âge minimum et } \\
\text { maximum requis }\end{array}$ & $14-28$ & $14-30$ & $14-30$ \\
\hline Date de création & 1997 & 1998 & 1996 \\
\hline Siège & Rome & Milan & Rome \\
\hline Organisation antérieure & Pas d'organisation officielle & Pas d'organisation officielle & Fronte della Gioventù \\
\hline Organigramme & $\begin{array}{l}\text { Un secrétaire national puis } \\
\text { coordinateurs } \\
\text { régionaux/ provinciaux }\end{array}$ & $\begin{array}{l}\text { Un coordinateur fédéral puis } \\
\text { coordinateurs } \\
\text { régionaux/ provinciaux }\end{array}$ & $\begin{array}{l}\text { Un président national } \\
\text { puis secrétaires } \\
\text { régionaux/ provinciaux }\end{array}$ \\
\hline & / grandes villes/ sections & / sections & / sections \\
\hline $\begin{array}{l}\text { Présence sur le } \\
\text { territoire }\end{array}$ & Toutes les régions & Régions septentrionales & Toutes les régions \\
\hline $\begin{array}{l}\text { Organisations } \\
\text { étudiantes dans le } \\
\text { secondaire }\end{array}$ & Azione Studentesca & $\begin{array}{l}\text { Movimento Studentesco } \\
\text { Padano Federale }\end{array}$ & $\begin{array}{l}\text { Pas d'organisation } \\
\text { spécifique }\end{array}$ \\
\hline $\begin{array}{l}\text { Organisations } \\
\text { étudiantes dans le } \\
\text { supérieur }\end{array}$ & Azione Universitaria & $\begin{array}{c}\text { Movimento Universitario } \\
\text { Padano }\end{array}$ & Studenti per la Libertà \\
\hline
\end{tabular}

\footnotetext{
${ }^{3}$ Ilvo Diamanti, La Lega. Geografia, storia e sociologia di un nuovo soggetto politico, Rome, Donzelli, 1993.

${ }^{4}$ Emanuela Poli, Forza Italia. Strutture, leadership e radicamento territoriale, Bologne, Il Mulino, 2001.

${ }^{5}$ Christophe Bouillaud, « Les droites en Italie. La science politique italienne face à un objet renouvelé », Politix, 30, 1995, p. 151-167; Marc Lazar, L'Italie à la dérive, Paris, Perrin, 2006.

6 Jean-Louis Briquet, «Leçons d'une crise: l'affirmation de la droite italienne », dans Frédérique Matonti (dir.), La démobilisation politique, Paris, La Dispute, 2005, p. 229-250.

7 Annie Collovald, «Pour une sociologie des carrières morales des dévouements militants », dans L'humanitaire ou le management des dévouements. Enquête sur un militantisme de "solidarité internationale" en faveur du Tiers Monde, Rennes, PUR, 2002, p. 177-229.
} 
Stéphanie Dechezelles, « Des partis et des jeunes. Les droites juvéniles dans l'Italie de Berlusconi », Histoire@Politique. Politique, culture, société, N4, janvier-avril 2008, www.histoire-politique.fr

Après avoir présenté les attendus sociologiques de l'étude d'un tel objet et les principales problématiques méthodologiques qui lui sont liées, il sera temps de montrer dans les principales différences entre organisations de jeunesse dans les conditions de socialisation, les modalités d'engagement et la gestion du pouvoir. Cette hétérogénéité dans les modes d'investissement militant et dans l'exercice de responsabilités politiques s'articule à des spécificités culturelles propres à chaque groupe militant, qui impriment leur marque sur les pratiques et les trajectoires des jeunes engagés.

\section{Les jeunes militants des droites italiennes : de l'opportunité d'un objet aux moyens de l'étudier}

\section{Les attendus de l'étude des organisations partisanes juvéniles}

Contrairement à leurs aînés, les jeunes Italiens qui ont entre 15 et 30 ans au cours de la dernière décennie du $\mathrm{XX}^{\mathrm{e}}$ siècle se socialisent et se politisent dans une configuration partisane fortement évolutive. De même, ils vivent dans un contexte général de désaffection à l'égard du politique dans ses versions « conventionnelles », les partis ou les syndicats ${ }^{8}$. En dépit de taux d'adhésion partisane et syndicale plus élevés que dans d'autres pays européens ${ }^{9}$, et au-delà des disparités sociales entre individus, la grande majorité des jeunes Italiens subit ce climat de méfiance et de défiance à l'égard des élites politiques du pays. Ceux qui s'engagent malgré tout le font de plus en plus souvent au sein d'organisations associatives ou autogérées (collectifs, coordinations, forums) autour de projets liés à l'humanitaire, l'altermondialisme ou encore les loisirs.

Parce que l'engagement de type partisan ne constitue pas aujourd'hui pour les jeunes le mode privilégié de participation politique, parce que les partis ne sont pas véritablement considérés comme des institutions valorisantes et que la politisation passe par des canaux alternatifs (associations, altermondialisme, autogestion, mouvements sociaux...), l'étude de ceux qui le pratiquent malgré tout permet de comprendre par le revers les transformations à l'ouvre dans la sphère sociopolitique italienne. De même, parce qu'au cours des années 1990, les ancrages partisans locaux des partis, principalement la Democrazia Cristiana sur les terres «blanches » (Lombardie, Vénétie, etc.) et le Partito Comunista sur les terres « rouges » (EmilieRomagne, Toscane, Marches, etc.) subissent de plein fouet la disparition de leur parti principal, il est important d'observer comment les jeunes, dépourvus de ce canal d’affiliation et d’adhésion politique, décident de s'investir en politique quand même.

\footnotetext{
8 Olivier Galland, Sociologie de la jeunesse, Paris, Armand Colin, 1991.

9 Susan E. Scarrow («Parties Without Members ? Party Organization in a Changing Electoral Environment », dans Russell J. Dalton et Martin P. Wattenberg (dir.), Parties Without Partisans, Oxford, Oxford University Press, 2000, p. 79-101) montre que d'une façon générale, les Italiens adhèrent en plus grand nombre que les autres habitants des pays de l'OCDE à un parti politique dans les années 1950-1980. Plus récemment, Anne Muxel et Marlaine Cacouault (dans Les jeunes d'Europe du Sud et la politique. Une enquête comparative France, Italie, Espagne, Paris, L'Harmattan, 2001, p. 1819) ont constaté qu'au regard de leurs homologues français et espagnols, «les jeunes Italiens se distinguent par un taux d'adhésion » aux syndicats et aux partis politiques « nettement supérieur, et par une plus grande activité dans le cadre des campagnes électorales ».
} 
Stéphanie Dechezelles, « Des partis et des jeunes. Les droites juvéniles dans l'Italie de Berlusconi », Histoire@Politique. Politique, culture, société, N4, janvier-avril 2008, www.histoire-politique.fr

Si pour tout un chacun, s'engager en politique ne constitue un acte ni anodin ni anecdotique, car l'individu y joue une partie importante de son identité sociale, pour les jeunes cette activité se révèle encore plus problématique. Parce que cette dernière s'inscrit dans un contexte de forte délégitimation des élites dirigeantes et parce qu'il expose en outre à un seuil minimal de publicité, l'engagement politique ne recouvre pas seulement une dimension marginale de la biographie d'un individu. Se situant à la jonction de la sphère privée et de la sphère publique, dont les logiques de fonctionnement diffèrent, l'engagement militant impose une négociation identitaire permanente et complexe de la part d'individus en phase d'incertitudes multiples (affective, familiale, professionnelle...) ${ }^{10}$. Comprendre comment cette négociation prend forme peut ainsi apporter des éléments de compréhension plus vastes sur la manière dont se font (et se défont) des collectifs militants.

Les jeunes engagés en politique nous semblent donc en mesure de servir de révélateurs pertinents pour saisir un fait social (ici l'engagement au sein de partis politiques) dans un contexte en profonde mutation. C'est pourquoi nous avons « profité » de la victoire de la coalition italienne de «droite » en mai 2001 pour engager la recherche et tester ainsi ce qui peut subsister de pratiques anciennes et ce qui relève de l'innovation, ce qui est de l'ordre de la rémanence et de celui de la nouveauté. En particulier, en tant que viviers de dirigeants politiques ${ }^{11}$, les organisations de jeunesse permettent d'observer les processus de professionnalisation politique et les voies d'accès aux carrières politiques, à plus forte raison lorsqu'il s'agit de partis relativement récents ou n'ayant pas encore exercé de pouvoir au niveau national (en particulier les «droites » élues pour la première fois en 1994 mais demeurées à peine un an à la tête du pays). Une étude portant sur les jeunes militants des partis de « droite » comporte également l'avantage de compléter les connaissances sur ces organisations, qui pour la plupart demeuraient des « boîtes noires », rarement pénétrées par les enquêteurs ou les commentateurs. En outre, sortir de l'ombre les acteurs prenant part au jeu politique « sur le terrain » et non pas seulement dans les palais du pouvoir constitue un motif supplémentaire d'intérêt.

Enfin, l'étude des mouvements de jeunesse et des jeunes militants des partis permet de rompre avec une vision homogénéisante et donc, en quelque sorte, enchantée des organisations partisanes. En effet, ils participent de jeux et d'enjeux de pouvoir, ne serait-ce que pas la position de «dominés » ou de «prétendants » dans laquelle les placent les adultes du parti ${ }^{12}$. De même, en faisant eux aussi partie de courants ou en étant acteurs de luttes internes au parti, les jeunes militants appartiennent à de multiples composantes qui constituent ce que l'on objective sous le terme de « parti ».

\footnotetext{
10 Anne Muxel, «La participation politique des jeunes : soubresauts, fractures, ajustements », Revue française de science politique, 52 (5-6), 2002, p. 521-544.

11 Selon Ettore Recchi, les anciens membres des organisations de jeunesse représentent plus de $30 \%$ des parlementaires italiens élus en 1992 et 1994 ; toujours selon le même auteur, dans la première moitié des années 1990, plus de $70 \%$ des anciens jeunes cadres dirigeants des organisations de jeunesse figurent dans le Who's who ? en tant que détenteurs de positions politiques. Cf. Ettore Recchi, « L'entrée en politique des jeunes Italiens : modèles explicatifs de l'adhésion partisane », Revue française de science politique, 51(1-2), 2001, p. 155-174.

12 Pierre Bourdieu, «La jeunesse n'est qu'un mot », dans Questions de sociologie, Paris, Minuit, 1984, p. 143-154.
} 
Stéphanie Dechezelles, « Des partis et des jeunes. Les droites juvéniles dans l'Italie de Berlusconi », Histoire@Politique. Politique, culture, société, N4, janvier-avril 2008, www.histoire-politique.fr

\section{Un protocole d'enquête adapté à la population militante juvénile}

Travailler sur les représentations (ou « cultures militantes ») en même temps que sur les trajectoires de jeunes militants suppose de recueillir des supports diversifiés de propagande partisane : non seulement les affiches, tracts, programmes, sites Internet, fanzines, statuts mais aussi les oeuvres musicales, littéraires, artistiques mises en avant par les directions juvéniles ou encore la littérature « grise » produite par des intellectuels, des hommes politiques ou d'anciens militants à propos du parti. Il importait également de rassembler des éléments d'observation in situ des conditions des pratiques militantes des jeunes ; à diverses occasions, il a ainsi été possible d'assister à des meetings de campagne, à des fêtes de parti, à des réunions de section, des repas ou à des manifestations organisés par les sections juvéniles des différents partis étudiés. Ce matériau a été consigné sous forme de carnets de terrain, exploités de manière non systématique lors de la restitution des résultats finaux. Enfin, la production de résultats appelait un second type de sources indispensables. Il s'agissait d'entretiens réalisés en face-à-face auprès de jeunes engagés dans chacun des partis étudiés : 89 entretiens semi-directifs à caractère biographique ont été ainsi réalisés entre fin 2001 et fin 2002

Grâce à la proximité de l'âge et des conditions de vie (étudiante) entre enquêtrice et enquêtés, il a été relativement facile d'entrer en contact avec les acteurs sans que ces derniers ne ressentent un fossé statutaire ou trop d'intimidation face à une sollicitation et un exercice peu courants. Par ailleurs, malgré les problèmes liés à la rétrodiction comme la sélectivité des souvenirs, la hiérarchisation subjective des faits, l'hypersubjectivité13, auxquelles s'ajoutent les caractéristiques mémorielles propres à la catégorie d'âge de la population étudiée - la moindre expérience biographique, l'importance accordée aux événements les plus récents, l'immersion dans la période où se vivent les « événements conducteurs » qui influeront sur le comportement futur ${ }^{14}$-, les entretiens biographiques ont permis l'accès aux deux ordres, objectif et subjectif, du discours. Les récits ainsi obtenus sur sollicitation du chercheur fournissaient à la fois un contenu informatif et un contenu narratif, renseignant de manière également appréciable sur les espaces, les histoires et les contextes d'expérience sociale tout en n'occultant pas la dimension émotionnelle ou affective. Les récits de vie des enquêtés permettaient aussi de reconstruire des trajectoires et de porter un regard diachronique sur l'objet de recherche, alors même que le suivi longitudinal des enquêtés s'avérait impossible du fait du manque de moyens matériels à disposition.

Une fois ces éléments méthodologiques circonscrits, il reste à présenter plus précisément les dimensions idéologiques et militantes des organisations de jeunesse étudiées et de comprendre ce qui fonde leur rapport singulier au militantisme et à l'exercice du pouvoir.

\footnotetext{
13 Olivier Fillieule, « Propositions pour une analyse processuelle de l'engagement individuel », Revue française de science politique, 51 (1-2), 2001, p. 199-217.

14 Olivier Ihl, « Socialisation et événements politiques », Revue française de science politique, 52 (2-3), 2002, p. 125-144.
} 
Stéphanie Dechezelles, « Des partis et des jeunes. Les droites juvéniles dans l'Italie de Berlusconi », Histoire@Politique. Politique, culture, société, N4, janvier-avril 2008, www.histoire-politique.fr

\section{Des modes d'engagement et d'exercice du pouvoir contrastés}

Après deux décennies de violence politique intense entre la fin des années 1960 et le début des années $1980^{15}$ —la période est d'ailleurs nommée «Années de Plomb» afin d'évoquer la chape de violence pesant sur la politique italienne - , la jeunesse militante des années 1990, de « gauche » et de « droite », voit les affrontements entre groupuscules ou fractions partisanes diminuer en nombre et en intensité. Des affrontements persistent entre les membres des structures occupées et autogérées, les Centri sociali, et les membres de groupes néo-fascistes, mais les morts au cours de combat se réduisent drastiquement dès les premières années 1980. En s'euphémisant, la violence physique se transforme soit en attribut des glorieux aînés tombés, comme leurs ascendants fascistes, pour la cause (chez les jeunes d'Alleanza Nazionale), soit un contre-modèle répulsif de comportement militant (chez les jeunes de Forza Italia et de la Lega Nord).

\section{Une hétérogénéité politique et militante}

Comme dans d'autres pays, les différentes «droites » juvéniles en Italie se caractérisent par une histoire, une idéologie, une culture, des modes d'action et une sociologie fortement contrastés. Celle qui peut se prévaloir del'héritage le plus ancien est sans conteste Alleanza Nazionale qui compte un mouvement de jeunesse important et structuré, Azione Giovani, en plus de deux grandes organisations syndicales (Azione Studentesca pour le secondaire et Azione Universitaria pour le supérieur). Née d'une refonte du parti néo-fasciste, le Movimento Sociale Italiano, lui-même reconstitué sur les cendres du Partito Nazionale Fascista à peine la guerre terminée (dès 1946 apparaissent les premières candidatures), Alleanza Nazionale constitue le pôle national et nationaliste des «droites » italiennes. Ayant en partie abandonné la référence au fascisme comme modèle de régime sous l'impulsion de son leader Gianfranco Fini, le parti est très fort électoralement dans le Sud de la péninsule, le Mezzogiorno, là où la nostalgie du régime mussolinien est encore vivace. Il s'agit d'un parti de masse et de militants, fortement hiérarchisé et implanté sur tout le territoire italien. Malgré une prédominance des cadres et élus méridionaux, le parti connaît des succès importants dans le Nord de la péninsule au cours des années 1990, notamment du fait de sa légitimation par Silvio Berlusconi qui lui propose à plusieurs reprises (1994, 1996, 2001 et 2006) une alliance électorale victorieuse.

La culture du mouvement de jeunesse d'Alleanza Nazionale, Azione Giovani, s'inspire très largement de celle du Fronte della Gioventù, organisation de jeunesse elle-même du Movimento Sociale Italiano. Elle se caractérise par la référence prégnante à l'héritage fasciste en tant que mouvement, la défense du modèle de régime fasciste sur la base de la République sociale italienne (régime fantoche aux mains des nazis de septembre 1943 à avril 1945, autrement appelé « République de Salò » du nom de la localité sur les rives du lac de Garde où ce régime s'installe), la glorification de héros de la cause, l'exhortation à la nostalgie et aux faits d'armes militants n'excluant pas les combats physiques avec les adversaires d'« extrême

${ }^{15}$ Isabelle Sommier, La violence politique et son deuil. L'après 68 en France et en Italie, Rennes, PUR, 1998. 
Stéphanie Dechezelles, « Des partis et des jeunes. Les droites juvéniles dans l'Italie de Berlusconi », Histoire@Politique. Politique, culture, société, N4, janvier-avril 2008, www.histoire-politique.fr

gauche ». En interne, la discipline et le sacrifice de soi fondent la hiérarchie : les plus méritants au regard des critères partisans sont récompensés selon des règles d'avancement très codifiées. Le jeune militant est appelé à se former et à se cultiver par les réunions, événements, commémorations prescrits par le groupe. Une attention spécifique est portée à la formation en matière historique, mais en vue d'une critique des lectures académiques et/ ou officielles de certains épisodes ou périodes (batailles de la Seconde Guerre mondiale, régime fasciste, Libération du pays par les Alliés et des terres dalmato-slovènes par l'armée yougoslave, Années de Plomb). L'une des activités des jeunes militants consiste ainsi à rédiger et diffuser des contre-manuels d'histoire à destination des lycéens et des étudiants, qui relèvent les extraits jugés factieux des ouvrages couramment utilisés dans l'enseignement secondaire et supérieur.

Les jeunes militants d'Alleanza Nazionale sont également incités à rédiger des tracts ou organiser des commémorations en l'honneur de ceux que l'organisation considère comme «ses » morts : militaires de certains corps d'armée durant le fascisme, compatriotes civils tués lors des combats dans le Nord-Est de la péninsule, jeunes militants décédés au cours de combats avec les adversaires d'« extrême gauche » durant les années 1970-1980. Par ailleurs, les sections juvéniles mettent en place des excursions à Predappio, village natal de Benito Mussolini dans lequel se trouve le caveau familial : se rendre sur la tombe du « Duce » ou, mieux, en effectuer une garde silencieuse pendant plusieurs heures constituent de véritables rétributions pour de jeunes militants socialisés dans l'admiration du chef.

Mais loin de ne relever que de l'apprentissage militant, les relectures de l'Histoire répondent à un véritable légendaire familial et à un substrat biographique. En effet, nombreux sont les jeunes de cette organisation qui peuvent revendiquer un ou plusieurs grands-parents/parents comme membre(s) du Partito Nazionale Fascista et/ou du Movimento Sociale Italiano. Au sein d'Alleanza Nazionale, le ressort de l'identification repose sur la continuité mémorielle et la perpétuation intergénérationnelle qui impliquent la célébration d'une époque bénie à laquelle les militants peuvent s'identifier, mais aussi rendre aux « anciens » l'hommage qu'ils méritent. C'est pourquoi la perpétuation et le souvenir font donc partie des devoirs imprescriptibles de chaque militant, et facilités par la transmission précise d'une histoire familiale, censée être peuplée d'êtres courageux et glorieux dont ils se perçoivent les héritiers. Mais, faisant appel à des épisodes ou des personnages fortement stigmatisés, les jeunes d'Alleanza Nazionale sont contraints d'utiliser un univers symbolique alternatif, leur permettant d'y puiser des références positives, en particulier la littérature fantastique, comme Le Seigneur des Anneaux de J.R.R. Tolkien. Utilisée par le Fronte della Gioventù dès les années 1970 et inspirée par la mythologie nord-européenne à laquelle se réfèrent souvent les mouvements d'inspiration fasciste, cette œuvre est littéralement appropriée par les jeunes du parti comme transposition dans un univers imaginaire des rapports qu'eux-mêmes, gentils Hobbits (petits personnages innocents, aux grands pieds et oreilles pointues), entretiendraient avec le Mordor (empire du Mal).

L'organisation de jeunesse de la Lega Nord n'apparaît que tardivement (19961997) par rapport au parti qui est fondé en 1990 lorsque s'unissent les diverses ligues régionales (Ligues lombarde, vénète, piémontaise, ligure, émilienne, frioulane, toscane) nées elles entre 1979 et le début des années 1980. D’abord fortement régionaliste et anti-méridionale, la Lega Nord se transforme progressivement en parti 
Stéphanie Dechezelles, « Des partis et des jeunes. Les droites juvéniles dans l'Italie de Berlusconi », Histoire@Politique. Politique, culture, société, N4, janvier-avril 2008, www.histoire-politique.fr

indépendant (pour la Sécession du Nord), anti-européen, homophobe et xénophobe. Les références, dans le matériel de propagande ou lors d'événements organisés par le parti, à des ancêtres celtes, barbares frustes opposés à la Rome impériale sont censés galvaniser les militants d'aujourd'hui pour défendre la Padanie contre la voracité de Rome la voleuse, les appétits des immigrés méridionaux mafieux et les immigrés extracommunautaires (i.e. non-européens). La plaine du Pô (la Padania) est ainsi construite en référence identitaire omniprésente d'un « âge d'or » à reconquérir : territoire des ancêtres barbares d'origine celte ou proto-vénète (Âges du Bronze et du Fer), opposés durant des siècles à Rome, il est surinvesti par les idéologues du parti, le leader et les militants, en particulier les jeunes qui y voient là une grille de lecture appropriée à leur situation présente. L'histoire mythique de ce pays imaginaire fait ainsi l'objet d'efforts importants d'《 invention de la tradition »16. Ce travail passe par exemple par la publication de nombreux articles et ouvrages d'amateurs en archéologie, histoire locale, langues anciennes cherchant à multiplier les preuves attestant de la «réalité » de la civilisation padane. Des Ligues communales médiévales (le nom même du parti évoque la Lega lombarda, union des communes libres de Lombardie et de la province véronaise contre les velléités de Frédéric I ${ }^{\mathrm{er}} \mathrm{de}$ Hohenstaufen en 1167) à la Sérénissime République de Venise, des « barbares » celtes aux chevaliers en armure, du pré-christianisme à la Réforme calviniste, tout est bon pour faire partie d'une histoire mythifiée dont l'objectif est aussi de prouver l'indéfectible différence ethnique et, par extension, culturelle, entre les peuples du Nord, laborieux et honnêtes, des peuples du Sud, colonisateurs, fainéants et corrompus, expliquant par une pirouette historiographique les prouesses actuelles des districts industriels du Nord et le retard du Mezzogiorno. CQFD.

Pour cela, il leur faut aussi opérer une contestation forte de l'histoire officielle et académique dans leurs divers canaux (bulletins, télévision et quotidien du parti), en particulier celle touchant au processus d'unité italienne. Ce faisant, ils se placent du côté de la contre-Histoire, alternant entre celle des peuples opprimés et celle des peuples libres, en tous cas à l'opposé de la Romanité, souvent source de fierté nationale en Italie. Les jeunes investissent alors une symbolique et une sociabilité militantes liées à la fois à la celtitude et à la chevalerie (tournois, fêtes, concerts et jeux de rôle costumés). Le film hollywoodien Braveheart ${ }^{17}$ mettant en scène le chef des tribus écossaises William Wallace en guerre contre le roi anglais Edward Ier est utilisé pour l'iconographie et les slogans du Movimento Giovani Padani. Mais ce qu'on taxe parfois de folklore ou d'idiotie léghiste fonctionne parce que cette fantaisie politique ne rencontre pas d'obstacle de la part des individus qui y croient. En effet, les jeunes militants de la Lega Nord ont en commun de présenter une mémoire familiale très lacunaire et surtout centrée sur les souffrances infligées par la « Grande Histoire » sur la vie quotidienne de leurs ancêtres, les « petits », les « sans grades », les «paysans », manipulés à leur guise par «les Puissants». La Padanie semble remplir le vide mémoriel des jeunes militants, en le peuplant d'êtres et d'histoires fabuleux. Cela rend donc possible l'investissement d'énergies militantes juvéniles autour de mythologies positives des origines, et peut en partie expliquer pourquoi l'admiration et la fidélité envers le leader incontesté et incontestable, Umberto Bossi,

16 Eric Hobsbawm et Thomas Ranger (dir.), The Invention of Tradition, Cambridge, Cambridge University Press, 1993.

${ }^{17}$ Le film est réalisé en 1995 par Mel Gibson qui y tient le rôle titre. 
Stéphanie Dechezelles, « Des partis et des jeunes. Les droites juvéniles dans l'Italie de Berlusconi », Histoire@Politique. Politique, culture, société, N4, janvier-avril 2008, www.histoire-politique.fr

sont autant mises en avant au sein de l'organisation de jeunesse. Aucune critique ni aucune faction dissidente n'est tolérée, sous peine d'expulsion.

Les carrières des jeunes sont donc soumises aux décisions prises au sommet du parti et, de là, sujettes à de grandes variations : ainsi la vague de défections qui a secoué la Lega Nord en 1997 a poussé Umberto Bossi à accorder des postes d'éligibilité aux élections locales des personnes en qui il pensait pouvoir avoir totale confiance : les jeunes et les femmes sans ressources politiques ou notabiliaires trop importantes. L'engagement dans la Lega Nord est appelé par l'organisation ellemême à n'être pas " politique », synonyme d'intéressement, d'opportunisme ou de carriérisme ${ }^{18}$. Il est demandé aux jeunes de pratiquer plutôt un don de soi, un acte bénévole au service de leurs frères du Nord, opprimés par l'État italien. La formation militante des jeunes compte alors moins que la pratique et l'organisation d'événements censés être apolitiques : tournois sportifs, concerts et fêtes locales (à base de polenta, de bière ou d'hydromel), élection de Miss Padanie, etc.

Initialement prévue (fin 1993-début 1994) pour n'être qu'un ensemble de comités électifs soutenant lors des votations Silvio Berlusconi, son leader et créateur, Forza Italia concède toutefois la création d'une organisation aux jeunes adhérents du parti dans la seconde moitié des années 1990. Regroupant de nombreux exdémocrates chrétiens et socialistes, Forza Italia développe une idéologie d'inspiration néo-libérale, atlantiste et conservatrice. Viscéralement opposés au Partito Comunista Italiano et à ses héritiers (Democratici di Sinistra et Rifondazione Comunista surtout), les membres de Forza Italia en rejettent également le modèle en matière de militantisme. L'engagement sacrificiel et total est décrié au profit d'un activisme plus individuel et explicitement carriériste. Les recrutements se font principalement au sein des couches moyennes, professions libérales, dirigeants des PME/ PMI, et plutôt auprès des populations urbaines du Nord du pays.

Fondant son discours d'auto-légitimation lors de son entrée officielle en politique sur sa supposée « nouveauté19 » ainsi que sur la radicale rupture souhaitée avec la Première République, ses élus accusés de corruption et ses pratiques troubles, Silvio Berlusconi impose également un style rhétorique volontairement axé sur l'absence de références historiques. Le temps utilisé dans les documents et les discours du parti et de son organisation de jeunesse est celui du présent, indéfini et infini à la fois. Seraient ainsi venues l'époque du pragmatisme et la fin des idéologies utopistes fondées sur des références au passé (fascisme) ou à un futur radieux espéré (communisme). De la même façon, l'iconographie est relativement pauvre et presque exclusivement organisée autour de portraits du leader ${ }^{20}$, ce qui correspond en partie à une traduction sur le plan de l'image d'un présupposé fort de l'idéologie du parti : la

18 Le modèle d'engagement à la Lega Nord correspond au modèle développé par Daniel Gaxie dans « Economie des partis et rétributions du militantisme », Revue française de science politique, 27 (1), 1977, p. 123-154 puis dans « Rétributions du militantisme et paradoxes de l'action collective », Revue suisse de science politique, 11(1), 2005, p. 157-188.

19 Nouveau candidat aux élections législatives de mars 1994 et leader d'un nouveau parti, Silvio Berlusconi est loin d'être un novice en politique. Aussi bien par ses réseaux personnels, ses liens politico-professionnels avec les principaux politiques d'envergure nationale, notamment le secrétaire du Partito Socialista Italiano Bettino Craxi, ainsi qu'avec de nombreux élus locaux dans tout le pays, en particulier en Lombardie et dans le Sud et les Iles, Silvio Berlusconi ne fait pas vraiment figure d'outsider inconnu.

20 Luciano Cheles, «L'image au pouvoir. Les portraits de Berlusconi », Vingtième Siècle. Revue d'histoire, 80, 2003, p. 112-122. 
Stéphanie Dechezelles, « Des partis et des jeunes. Les droites juvéniles dans l'Italie de Berlusconi », Histoire@Politique. Politique, culture, société, N4 , janvier-avril 2008, www.histoire-politique.fr

construction autonome du sujet dépouillé de toute attache antérieure et détaché de toute contingence extérieure. C'est bien ici la figure du « self-made man » qui est appropriée par les jeunes militants et qui transpire de leurs propres récits biographiques et d'engagement. Géré à partir des canons du management entrepreneurial à la façon des entreprises du groupe Fininvest (empire berlusconien), le parti mise également beaucoup sur l'instrumentalisation des techniques modernes de propagande et les techniques du marketing politique.

Dans ce parti et son organisation de jeunesse, les ressources individuelles comptent beaucoup dans les possibilités d'accès à l'éligibilité et les opportunités d'avancement politique. Plus que de militants activistes, Forza Italia et Forza Italia Giovani sont ainsi composées de petits entrepreneurs individuels de leur propre carrière. Les activités collectives sont relativement rares et visent le plus souvent à soutenir le leader (ou le leader local) lors de congrès ou de manifestations spectaculaires; elles s'accompagnent d'une débauche de moyens et d'objets à caractère publicitaire : drapeaux, pin's, cravates, casquettes, montres à l'effigie de Silvio Berlusconi ou aux couleurs du parti (bleu azur). Plus que la formation ou la socialisation internes, c'est la multiplication des inscriptions sociales, professionnelles, associatives qui est recommandée pour espérer faire carrière et se maintenir au pouvoir. L'engagement au sein de Forza Italia Giovani rend alors toute carrière suspendue aux ressources que chaque individu est en mesure de mobiliser de façon continue pour s'imposer face à d'autres concurrents.

\section{Un rapport différencié à l'exercice individuel et/ou collectif du pouvoir lors du second gouvernement Berlusconi (2001-2006)}

$\mathrm{Si}$ la forte centralisation et la domination exclusive d'Umberto Bossi sur la Lega Nord jusqu'à son accident cérébral de 2004 empêchent toute minorité ou courant interne de se constituer de manière durable au sein de ce parti, l'histoire d'Alleanza Nazionale, héritière du néo-fasciste Movimento Sociale Italiano ${ }^{21}$, est marquée quant à elle par l'opposition entre plusieurs courants auxquels s'affilient également les jeunes militants. Cette différence explique en partie l'autonomie de chacune des organisations de jeunesse par rapport au parti. De l'instrument de légitimation aux mains d'Umberto Bossi à la Lega Nord, au vivier de futurs cadres et candidats du parti à Alleanza Nazionale, en passant par un entre-deux statutaire à Forza Italia, les jeunes militants des «droites » italiennes ne sont pas tous égaux dans le rapport qu'ils sont susceptibles d'entretenir avec leur parti. Dans ce cadre, c'est certainement l'organisation de jeunesse d'Alleanza Nazionale qui bénéficie de la plus grande marge d'autonomie, en dépit de divisions au sein des courants internes entre la ligne conservatrice du secrétaire national, Gianfranco Fini, et une ligne plus socialo-fasciste à laquelle adhèrent de nombreux jeunes. C'est également Azione Giovani qui constitue le vivier de futurs professionnels politiques et le lieu

\footnotetext{
${ }^{21}$ Parti politique recréé en décembre 1946, c'est-à-dire au lendemain de la fin de la Seconde Guerre mondiale et de la disparition de Benito Mussolini, par d'anciens dignitaires de la Repubblica Sociale Italiana. Ses principaux dirigeants sont Arturo Michelini et Giorgio Almirante, qui en fut secrétaire de 1946 à 1950 puis de 1969 à 1987 (un an avant sa mort). C'est par la suite son dauphin, Gianfranco Fini, qui est élu et qui reste jusqu'à aujourd’hui le secrétaire du parti (transformé en Alleanza Nazionale donc), après un bref intermède de 1990 à 1991 durant lequel c'est le très contesté social-fasciste Pino Rauti qui est élu à cette charge.
} 
Stéphanie Dechezelles, « Des partis et des jeunes. Les droites juvéniles dans l'Italie de Berlusconi », Histoire@Politique. Politique, culture, société, N4 , janvier-avril 2008, www.histoire-politique.fr

d'apprentissage du métier politique ${ }^{22}$, au regard des deux autres organisations qui soit bénéficient de moindres moyens logistiques, matériels et humains, soit sont assujetties d'abord à l'organisation adulte en lui fournissant à faible coût des « petites mains » militantes (affichage, tractage lors des campagnes électorales notamment). Malgré tout, les différences dans le rapport au pouvoir de ces différentes organisations de jeunesse de droite en Italie ne proviennent pas seulement de la place qu'elles occupent au regard de l'organisation «adulte», mais aussi des transformations qui ont marqué cette dernière et des évolutions en termes idéologiques.

La position de dominés ou, du moins, de prétendants dans laquelle se trouvent les jeunes des organisations partisanes les oblige à adopter une posture de défenseurs loyaux de la pureté de la ligne du parti ${ }^{23}$. Cela se vérifie surtout dans les partis fortement idéologisés, ou ayant une histoire longue de choix et de références idéologiques tranchées. De ce point de vue, les organisations de jeunesse de la Lega Nord mais plus encore Alleanza Nazionale se caractérisent par l'ambition de constituer les gardiens inflexibles du corpus idéologique originel, ou du moins celui désigné comme tel. Or, confrontés - plus souvent de force que de gré - aux mutations des partis (institutionnalisation, professionnalisation, renouvellement générationnel, flux de militants et d'élus...), les jeunes militants à la « base » interprètent souvent ces changements comme une perte ou un affadissement des valeurs sur lesquelles ils avaient fondé le sens de leur engagement: gratuité du dévouement, plaisir d'être ensemble, entre soi, solidarité et camaraderie...).

L'expérience relativement inédite du pouvoir par le parti au sein de coalitions gouvernementales ou locales ainsi que, pour certains, l'exercice d'un mandat au sein d'une assemblée locale, sont d'autres sources de dissonances. Ces dernières sont dues notamment à la nécessité de passer d'une culture de l'opposition à une culture de majorité gouvernante, au devoir de respecter des procédures routinisées de l'administration, à l'obligation de composer avec des alliés aux positions divergentes. Mais c'est surtout l'immixtion au sein de l'endocratie partisane de « recyclés » de l'ancien système politique ou d'anciens partis délégitimés de la Première République (Democrazia Cristiana et Partito Socialista en tête) ayant profité des succès des partis de «droite » et de la fin du cycle des procès de Mani pulite pour réintégrer le jeu politique, qui a entraîné des critiques de la part des jeunes militants. Beaucoup ont interprété ces arrivées comme du pur opportunisme mais aussi comme une trahison consentie, sinon orchestrée, par leurs propres dirigeants. Mais fortement incités à participer aux élections des représentants étudiants, dans l'enseignement secondaire comme dans le supérieur où ils font précocement leurs premières armes, les jeunes militants d'Alleanza Nazionale montrent une plus grande capacité à gérer ces flux de « nouveaux » militants que ceux de la Lega Nord, qui se tiennent plus à l'écart des mandats de représentants scolaires. Engagés dans un parti dont la culture militante valorise la réussite personnelle et le carriérisme, ces arrivées exogènes de nouveaux membres ont provoqué malgré tout déceptions et rancœurs chez certains jeunes de Forza Italia misant sur une carrière politique.

\footnotetext{
${ }^{22}$ La plupart des responsables nationaux actuels du parti sont d'anciens responsables du Fronte della Gioventù, à commencer par Gianfranco Fini mais aussi Ignazio La Russa, Francesco Storace, Gianni Alemanno, Antonio Gasparri...

23 Sur les trois types de comportement possible au sein d'une organisation en cas de situation insatisfaisante pour un individu (la fidélité loyalty, la critique voice et la défection exit), voir Albert $\mathrm{O}$. Hirschmann, Défection et prise de parole, Paris, Fayard, 1995.
} 
Stéphanie Dechezelles, « Des partis et des jeunes. Les droites juvéniles dans l'Italie de Berlusconi », Histoire@Politique. Politique, culture, société, N4, janvier-avril 2008, www.histoire-politique.fr

L'étude des modes d'engagement et des trajectoires de jeunes au sein des partis permet à la fois de dépasser une vision uniforme des organisations partisanes et de mettre au jour les procédés de construction et d'appropriation d'une culture commune. Aborder un parti par l'une de ses composantes, ici les organisations de jeunesse, et sa population spécifique offre également de comprendre la nature et l'intensité des enjeux de pouvoir internes. Se démarquant des « adultes » sans pour autant s'en différencier totalement, les jeunes des partis sont intéressants à étudier afin de saisir les types de trajectoires valorisées par le groupe et les étapes de la professionnalisation politique spécifiques à chaque organisation. Il nous est apparu que l'étude des jeunesses militantes de «droite » et d' « extrême droite » en Italie permettait également de saisir le poids et le rôle des représentations du passé chez les jeunes engagés en politique aujourd'hui. Elle a notamment conduit à mesurer la pérennité des oppositions dialectiques «communistes》 versus «fascistes », oppositions entretenues par les organisations elles-mêmes, dans les cultures et les discours militants.

Réalisée au cours du second gouvernement Berlusconi (2001-2006), cette enquête nécessiterait sans doute des prolongements. En effet, il serait intéressant de mesurer ce qui a changé au sein des organisations étudiées, à l'heure (début 2008) où les «droites » italiennes sont retournées dans l'opposition et où le système partisan se trouve en profond remaniement: fusion de plusieurs partis de "gauche-centre gauche » (Democratici di Sinistra, Margherita et divers petits partis) au sein du Partito Democratico mené par l'ancien maire de Rome Walter Valtroni et refondation sous la houlette de Silvio Berlusconi de Forza Italia en Partito del Popolo delle Libertà avec le Nuovo Partito Socialista Italiano et certains dirigeants d'un des héritiers démocrates-chrétiens, l’Unione dei Democratici Cristiani e di Centro.

\section{L'auteur}

Actuellement en contrat post-doc au Cracs (centre régional du Cereq) et chercheure associée à Spirit (UMR CNRS 5116) - université de Bordeaux-Sciences Po Bordeaux, Stéphanie Dechezelles est l'auteure d'une thèse de science politique portant sur les cultures militantes et le sens de l'engagement au sein des organisations de jeunesse des «droites » italiennes. Elle a notamment publié « Mémoires militantes, histoire nationale et émotion. Héritiers fascistes et orphelins padans en Italie», dans Christophe Traïni (dir.), S'émouvoir pour la cause. Sensibilisation et mobilisations collectives, Paris, Presses de Sciences Po (à paraître, 2008) et « Entre révolution et gestion. L'engagement des jeunes militant(e)s de la Ligue du Nord et d'Alliance Nationale face à l'expérience du pouvoir en Italie », in Philippe Poirier et Pascal Delwit (dir.), Les nouvelles droites au pouvoir en Europe, Bruxelles, Ed. de l'ULB, 2007, p. 225-246. Ses recherches portent aussi sur la gestion partisane des oppositions au processus d'européanisation en Italie, dans le cadre d'un projet ANR « Europopulisme - Reconfiguration des jeux politiques populistes dans l'espace politique européen ».

\section{Résumé}

Le délitement et le désaveu des organisations piliers de l'après-guerre (Democrazia Cristiana et Partito Socialista en tête), le lancement des procès pour corruption entre sphères politiques et économiques (grâce aux opérations de Mani pulite) et les stratégies de conquête du marché électoral par des acteurs alternatifs bouleversent le système partisan italien. C'est en particulier le cas à « droite » (Forza Italia, Alleanza Nazionale, Lega Nord). De ce fait, les jeunes militants de ces organisations accèdent ou, du moins, s'approchent de l'exercice de responsabilités. Issues de traditions idéologiques différentes et fondées sur des cultures militantes contrastées, ces organisations juvéniles se distinguent à la fois dans le type de formation politique proposée aux jeunes activistes et dans la manière de gérer l'obtention de mandats électifs. Après avoir 
Stéphanie Dechezelles, «Des partis et des jeunes. Les droites juvéniles dans l'Italie de Berlusconi », Histoire@Politique. Politique, culture, société, N4, janvier-avril 2008, www.histoire-politique.fr

présenté brièvement les conditions spécifiques d'une enquête sociologique auprès de cette population militante juvénile, l'article s'attache à montrer comment la culture militante en matière mémorielle, distillée par chaque organisation et appropriée par les jeunes, s'articule à une biographie individuelle et familiale, permettant de donner un sens à l'engagement présent au travers d'une lecture spécifique du rapport intime à l'Histoire.

Mots-clés : militantisme ; jeunesse ; extrême droite ; Italie ; culture politique.

\begin{abstract}
Decay and denial of the post-war pillar-organisations (first of all Democrazia Cristiana and Partito Socialista), trials against corruption inside political and economical activities (thanks to the Mani pulite investigations) and alternative actors who seek to conquer the electoral market : all these elements disrupt the Italian partisan system. It is particularly true on the right side (Forza Italia, Alleanza Nazionale and Lega Nord). Then, their young activists reach or, for the less, draw near to political responsibilities. Coming from different ideological traditions and based on contrasted activist culture, these youth organisations differentiate one from the others in two ways : the type of political education they offer to the young activist and the way they manage the elective gains. After a brief presentation of the specific conditions for a sociological research, the article aims to demonstrate how the political and militant culture, especially as memory is concerned, that produce every party and that the young militants are supposed to appropriate, joint with an individual and familial biography, that give a means at the present involvement through a specific link with History.
\end{abstract}

Key words : Militancy ; Youth ; Right Wing Parties ; Italy ; Political Culture. 